\title{
Three Simultaneous Superimposed Rotating System
}

\author{
Mukul Chandra Das ${ }^{1,2, *}$, Rampada Misra ${ }^{3}$ \\ ${ }^{1}$ Singhania University, Pacheri Bari - 333515, Jhunjhunu, Rajasthan, India \\ ${ }^{2}$ Satmile High School, Satmile - 721452, Contai, West Bengal, India \\ ${ }^{3}$ Department of Electronics, Vidyasagar University, Midnapore - 721102, West Bengal, India \\ *Email address: mukuldas.100@gmail.com
}

\begin{abstract}
In inertial system, co-ordinate transformation from one frame to another is possible by using Lorentz transformation matrix. But in non-inertial or rotating system it is not applicable by using Lorentz transformation matrix. In this paper, co-ordinate transformation from one frame to another in three simultaneous superimposed rotating systems has been introduced. This also leads to assume a picture of space-time geometry of same system.
\end{abstract}

Keywords: non-inertial system; co-ordinate transformation; space-time geometry

\section{THREE SIMULTANEOUS SUPERIMPOSED ROTATIONAL MOTION}

According to [1,2] A particle or an event may possess two simultaneous superimposed motions (i.e. either linear or rotational). Similarly a particle or an event may possess three simultaneous superimposed rotational motions. For clarity of three simultaneous superimposed rotational motions as well as three simultaneous superimposed spins, following [3], it is stated that frames $S$ and $S_{1}$ have both their $X$ axes aligned and $S_{1}$ is moving at an angular velocity $\omega_{1}$ about $X_{1}$ axis as observed by $S$.

The frame $S_{1}$ has another co-ordinate reference frame $S_{2}$, where $X_{2}$ axis of $S_{2}$, are rotated by an angle $\theta$ counter clockwise with respect to $S_{1}$ on $X_{1} Y_{1}$ plane. Frames $S_{2}$ and $S_{3}$ have both their $X$ axes aligned and $S_{3}$ is moving at an angular velocity $\omega_{2}$ about $X_{3}$ axis as observed by $S_{2}$. Similarly $S_{3}$ has another co-ordinate reference frame $S_{4}$, where $X_{3}$ axis of $S_{3}$, are rotated by an angle $\psi$ counter clockwise with respect to $S_{3}$ on $X_{3} Y_{3}$ plane. Frames $S_{4}$ and $S_{5}$ have both their $X$ axes aligned and $S_{5}$ is moving at an angular velocity $\omega_{3}$ about $X_{5}$ axis as observed by $S_{4}$.

For the case when the origin of frames are same with respect to $S$ and the particle be at the origin of $S_{3}$, then it possesses three simultaneous superimposed spins with respect to frame $S$. Coordinate transformation matrix from $S$ to $S_{5}$ would be $P_{i j}=R_{y z\left(\omega_{3} t\right)} R_{x z(\psi)} R_{y z\left(\omega_{2} t\right)} R_{x y(\theta)} R_{y z\left(\omega_{1} t\right)}$ 
and $R_{y z\left(\omega_{i} t\right)}=\left(\begin{array}{cccc}1 & 0 & 0 & 0 \\ 0 & \cos \omega_{i} t & \sin \omega_{i} t & 0 \\ 0 & -\sin \omega_{i} t & \cos \omega_{i} t & 0 \\ 0 & 0 & 0 & 1\end{array}\right)$, where, $i=1,2,3$

Hence, we get the relations between co-ordinates of different frames $S, S_{1}, S_{2}, S_{3}, S_{4}$ and $S_{5}$ as stated below

$$
\begin{aligned}
& \left(\begin{array}{l}
x_{1} \\
y_{1} \\
z_{1} \\
t_{1}
\end{array}\right)=\left(\begin{array}{cccc}
1 & 0 & 0 & 0 \\
0 & \cos \omega_{1} t & \sin \omega_{1} t & 0 \\
0 & -\sin \omega_{1} t & \cos \omega_{1} t & 0 \\
0 & 0 & 0 & 1
\end{array}\right)\left(\begin{array}{l}
x \\
y \\
z \\
t
\end{array}\right), \quad\left(\begin{array}{l}
x_{2} \\
y_{2} \\
z_{2} \\
t_{2}
\end{array}\right)=\left(\begin{array}{cccc}
\cos \theta & \sin \theta & 0 & 0 \\
-\sin \theta & \cos \theta & 0 & 0 \\
0 & 0 & 1 & 0 \\
0 & 0 & 0 & 1
\end{array}\right)\left(\begin{array}{l}
x_{1} \\
y_{1} \\
z_{1} \\
t_{1}
\end{array}\right) \\
& \left(\begin{array}{l}
x_{3} \\
y_{3} \\
z_{3} \\
t_{3}
\end{array}\right)=\left(\begin{array}{cccc}
1 & 0 & 0 & 0 \\
0 & \cos \omega_{2} t & \sin \omega_{2} t & 0 \\
0 & -\sin \omega_{2} t & \cos \omega_{2} t & 0 \\
0 & 0 & 0 & 1
\end{array}\right)\left(\begin{array}{l}
x_{2} \\
y_{2} \\
z_{2} \\
t_{2}
\end{array}\right), \quad\left(\begin{array}{l}
x_{4} \\
y_{4} \\
z_{4} \\
t_{4}
\end{array}\right)=\left(\begin{array}{cccc}
\cos \psi & 0 & \sin \psi & 0 \\
0 & 1 & 0 & 0 \\
-\sin \psi & 0 & \cos \psi & 0 \\
0 & 0 & 0 & 1
\end{array}\right)\left(\begin{array}{l}
x_{3} \\
y_{3} \\
z_{3} \\
t_{3}
\end{array}\right), \\
& \left(\begin{array}{l}
x_{5} \\
y_{5} \\
z_{5} \\
t_{5}
\end{array}\right)=\left(\begin{array}{cccc}
1 & 0 & 0 & 0 \\
0 & \cos \omega_{3} t & \sin \omega_{3} t & 0 \\
0 & -\sin \omega_{3} t & \cos \omega_{3} t & 0 \\
0 & 0 & 0 & 1
\end{array}\right)\left(\begin{array}{l}
x_{4} \\
y_{4} \\
z_{4} \\
t_{4}
\end{array}\right)
\end{aligned}
$$

Therefore, the transformation matrix for coordinates of an event from $S$ to $S_{5}$ would be as shown below

$$
\begin{aligned}
& P_{i j}=R_{y z\left(\omega_{3} t\right)} R_{x z(\psi)} R_{y z\left(\omega_{2} t\right)} R_{x y(\theta)} R_{y z\left(\omega_{1} t\right)} \\
& \left(\begin{array}{cccc}
1 & 0 & 0 & 0 \\
0 & \cos \omega_{3} t & \sin \omega_{3} t & 0 \\
0 & -\sin \omega_{3} t & \cos \omega_{3} t & 0 \\
0 & 0 & 0 & 1
\end{array}\right)\left(\begin{array}{cccc}
\cos \psi & 0 & \sin \psi & 0 \\
0 & 1 & 0 & 0 \\
-\sin \psi & 0 & \cos \psi & 0 \\
0 & 0 & 0 & 1
\end{array}\right)\left(\begin{array}{cccc}
1 & 0 & 0 & 0 \\
0 & \cos \omega_{2} t & \sin \omega_{2} t & 0 \\
0 & -\sin \omega_{2} t & \cos \omega_{2} t & 0 \\
0 & 0 & 0 & 1
\end{array}\right)\left(\begin{array}{cccc}
\cos \theta & \sin \theta & 0 & 0 \\
-\sin \theta & \cos \theta & 0 & 0 \\
0 & 0 & 1 & 0 \\
0 & 0 & 0 & 1
\end{array}\right)\left(\begin{array}{cccc}
1 & 0 & 0 & 0 \\
0 & \cos \omega_{1} t & \sin \omega_{1} t & 0 \\
0 & -\sin \omega_{1} t & \cos \omega_{1} t & 0 \\
0 & 0 & 0 & 1
\end{array}\right)
\end{aligned}
$$




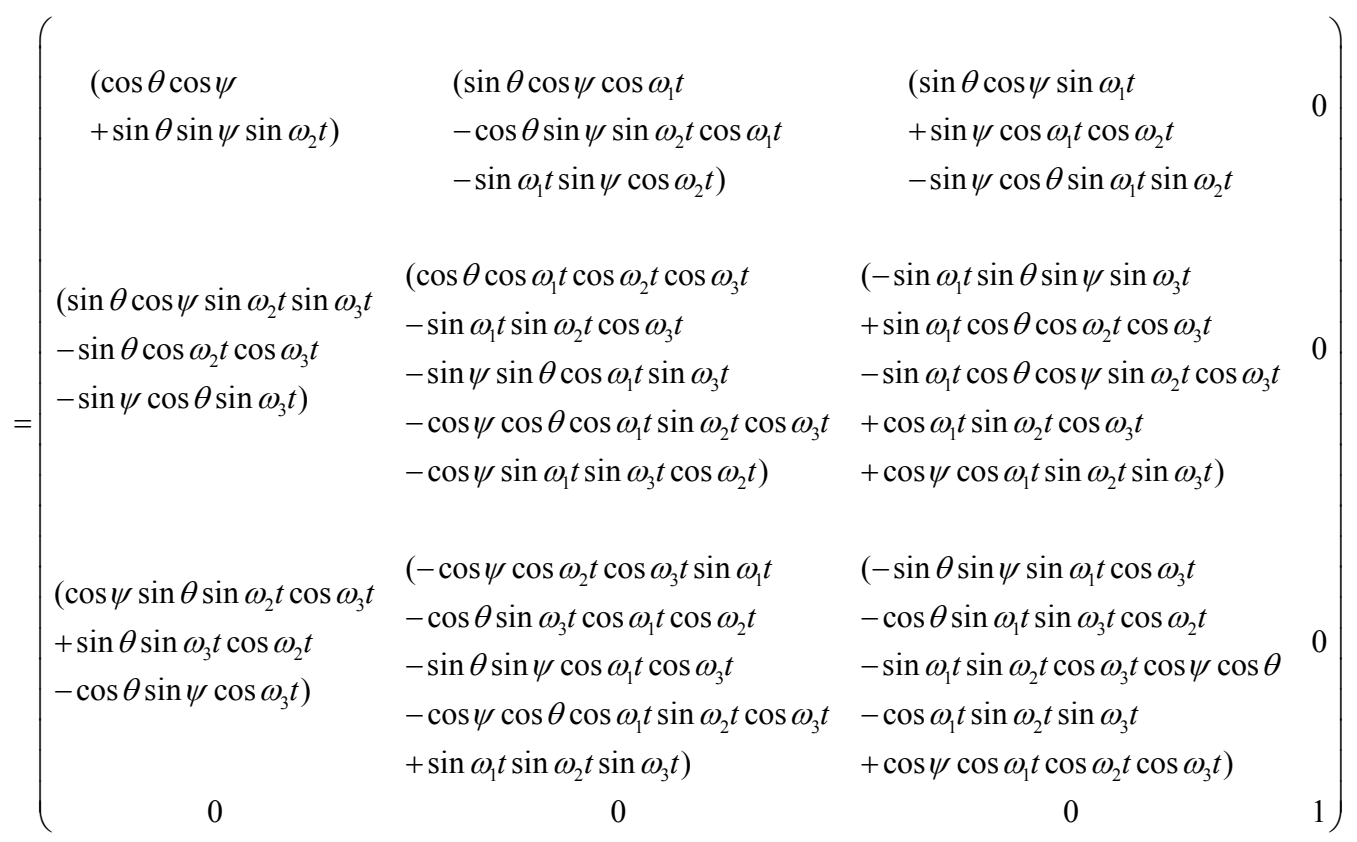

Similarly, coordinate transformation matrix of an event from $S_{5}$ to $S$ would be

$$
\begin{aligned}
& \bar{P}_{i j}=R_{y z\left(-\omega_{1} t\right)} R_{x y(-\theta)} R_{y z\left(-\omega_{2} t\right)} R_{x z(-\psi)} R_{y z\left(-\omega_{3} t\right)}= \\
& \left(\begin{array}{cccc}
1 & 0 & 0 & 0 \\
0 & \cos \omega_{1} t & -\sin \omega_{1} t & 0 \\
0 & \sin \omega_{1} t & \cos \omega_{1} t & 0 \\
0 & 0 & 0 & 1
\end{array}\right)\left(\begin{array}{cccc}
\cos \theta & -\sin \theta & 0 & 0 \\
\sin \theta & \cos \theta & 0 & 0 \\
0 & 0 & 1 & 0 \\
0 & 0 & 0 & 1
\end{array}\right)\left(\begin{array}{cccc}
1 & 0 & 0 & 0 \\
0 & \cos \omega_{2} t & -\sin \omega_{2} t & 0 \\
0 & \sin \omega_{2} t & \cos \omega_{2} t & 0 \\
0 & 0 & 0 & 1
\end{array}\right)\left(\begin{array}{cccc}
\cos \psi & 0 & -\sin \psi & 0 \\
0 & 1 & 0 & 0 \\
\sin \psi & 0 & \cos \psi & 0 \\
0 & 0 & 0 & 1
\end{array}\right)\left(\begin{array}{cccc}
1 & 0 & 0 & 0 \\
0 & \cos \omega_{3} t & -\sin \omega_{3} t & 0 \\
0 & \sin \omega_{3} t & \cos \omega_{3} t & 0 \\
0 & 0 & 0 & 1
\end{array}\right)
\end{aligned}
$$

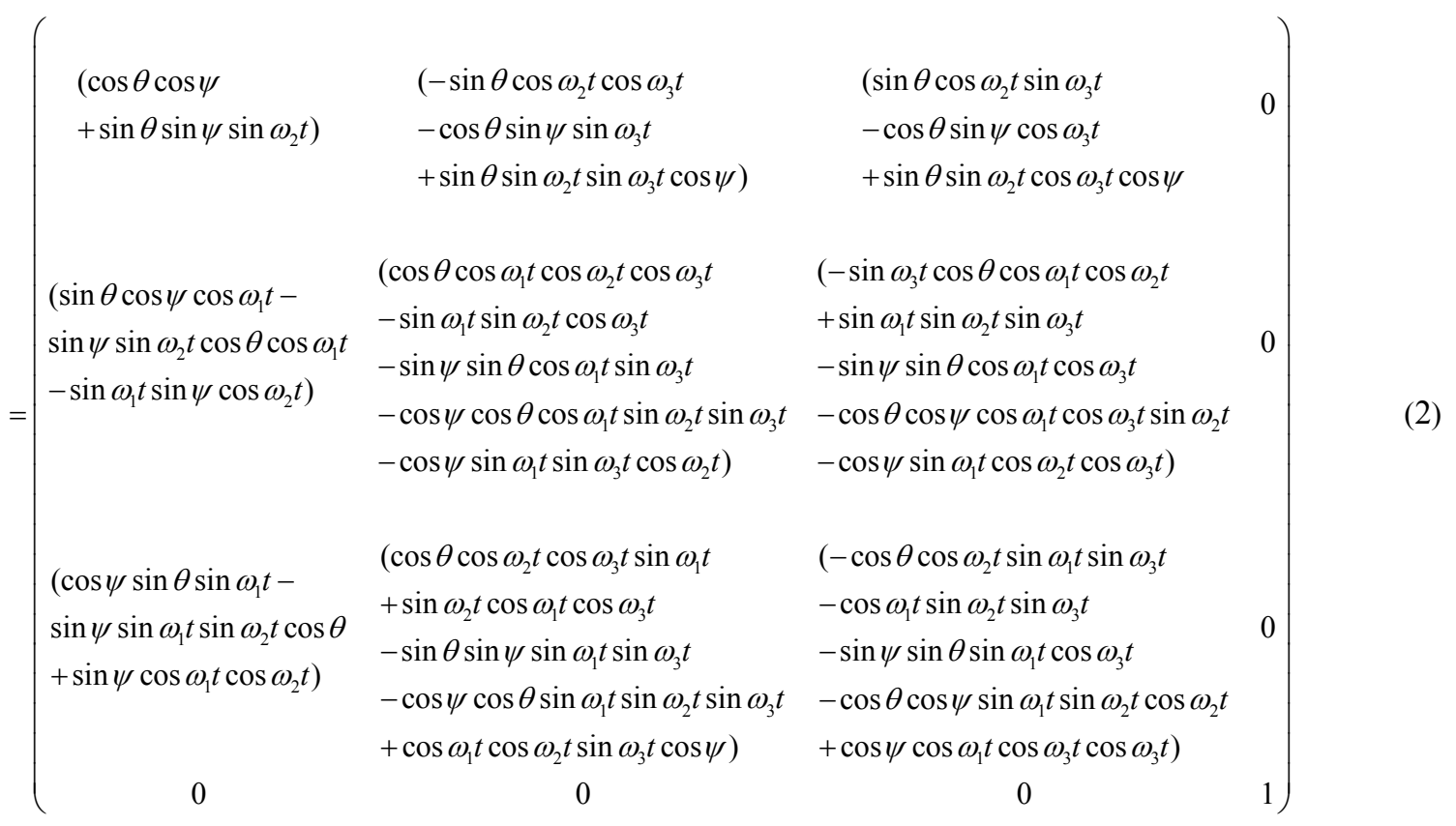


Now using (1) we get the relation between co-ordinates of frames $S$ and $S_{5}$ as shown below

$$
X_{5}\left(x_{5}, y_{5}, z_{5}, t_{5}\right)=P_{i j} X(x, y, z, t)
$$

Similarly using (2) we obtain the relation between co-ordinates of frames $S$ and $S_{5}$

$$
X(x, y, z, t)=\bar{P}_{i j} X_{5}\left(x_{5}, y_{5}, z_{5}, t_{5}\right)
$$

where, $X_{5}\left(x_{5}, y_{5}, z_{5}, t_{5}\right)=\left(\begin{array}{c}x_{5} \\ y_{5} \\ z_{5} \\ t_{5}\end{array}\right)$ and $\quad X(x, y, z, t)=\left(\begin{array}{c}x \\ y \\ z \\ t\end{array}\right)$

\section{SPACE-TIME GEOMETRY}

From (4) we obtain

$$
\begin{aligned}
& d x^{2}=\left(\bar{P}_{11} d x^{\prime}+\bar{P}_{12} d y^{\prime}+\bar{P}_{13} d z^{\prime}+\bar{P}_{14} d t^{\prime}\right)^{2} \\
& d y^{2}=\left(\bar{P}_{21} d x^{\prime}+\bar{P}_{22} d y^{\prime}+\bar{P}_{23} d z^{\prime}+\bar{P}_{24} d t^{\prime}\right)^{2} \\
& d z^{2}=\left(\bar{P}_{31} d x^{\prime}+\bar{P}_{32} d y^{\prime}+\bar{P}_{33} d z^{\prime}+\bar{P}_{34} d t^{\prime}\right)^{2} \\
& d t^{2}=\left(\bar{P}_{41} d x^{\prime}+\bar{P}_{42} d y^{\prime}+\bar{P}_{43} d z^{\prime}+\bar{P}_{44} d t^{\prime}\right)^{2}
\end{aligned}
$$

Now, we have Cartesian Co-ordinate geometry in flat space-time

$$
d s^{2}=-d t^{2}+d x^{2}+d y^{2}+d z^{2}
$$

Using (5) we obtain from (6), the space-time geometry of three simultaneous superimposed rotating system

$$
\begin{aligned}
d s^{2}= & A_{1} d x^{\prime 2}+A_{2} d y^{\prime 2}+A_{3} d z^{\prime 2}+A_{4} d t^{\prime 2}+2\left(\mathrm{~B}_{1} d x^{\prime} d y^{\prime}+B_{2} d x^{\prime} d z^{\prime}\right. \\
& \left.+B_{3} d x^{\prime} d t^{\prime}+B_{4} d y^{\prime} d z^{\prime}+B_{5} d y^{\prime} d t^{\prime}+B_{6} d z^{\prime} d t^{\prime}\right)
\end{aligned}
$$


where,

$$
\begin{array}{cc}
A_{1}=\bar{P}_{11}{ }^{2}+\bar{P}_{21}{ }^{2}+\bar{P}_{31}{ }^{2}-\bar{P}_{41}{ }^{2}, & A_{2}=\bar{P}_{12}{ }^{2}+\bar{P}_{22}{ }^{2}+\bar{P}_{32}{ }^{2}-\bar{P}_{42}{ }^{2} \\
A_{3}=\bar{P}_{13}{ }^{2}+\bar{P}_{23}{ }^{2}+\bar{P}_{33}{ }^{2}-\bar{P}_{43}{ }^{2}, & A_{4}=\bar{P}_{14}{ }^{2}+\bar{P}_{24}{ }^{2}+\bar{P}_{34}{ }^{2}-\bar{P}_{44}{ }^{2} \\
B_{1}=\bar{P}_{11} \bar{P}_{12}+\bar{P}_{21} \bar{P}_{22}+\bar{P}_{31} \bar{P}_{32}-\bar{P}_{41} \bar{P}_{42}, & B_{2}=\bar{P}_{11} \bar{P}_{13}+\bar{P}_{21} \bar{P}_{23}+\bar{P}_{31} \bar{P}_{33}-\bar{P}_{41} \bar{P}_{43} \\
\mathrm{~B}_{3}=\bar{P}_{11} \bar{P}_{14}+\bar{P}_{21} \bar{P}_{24}+\bar{P}_{31} \bar{P}_{34}-\bar{P}_{41} \bar{P}_{44}, & B_{4}=\bar{P}_{12} \bar{P}_{13}+\bar{P}_{22} \bar{P}_{23}+\bar{P}_{32} \bar{P}_{33}-\bar{P}_{42} \bar{P}_{43} \\
\mathrm{~B}_{5}=\bar{P}_{12} \bar{P}_{14}+\bar{P}_{22} \bar{P}_{24}+\bar{P}_{32} \bar{P}_{34}-\bar{P}_{42} \bar{P}_{44}, & \mathrm{~B}_{6}=\bar{P}_{13} \bar{P}_{14}+\bar{P}_{23} \bar{P}_{24}+\bar{P}_{33} \bar{P}_{34}-\bar{P}_{43} \bar{P}_{44}
\end{array}
$$

\section{CONCLUSION}

In this way any number of transformations or rotations may be considered. Also the process leads to conclude that a particle may possess more then one simultaneous superimposed spins with its mutual effects. Co-ordinate transformation in non-inertial systems would be done following the process as in the text.

\section{References}

[1] C. Iyer, G. M. Prabhu, Journal of Physical and Natural Sciences 1(2) (2007) 1-8.

[2] M. C. Das, R. Misra, International Letters of Chemistry, Physics and Astronomy 7(2) (2013) 73-84.

[3] M. C. Das, R. Misra, Advance Studies in Theoretical Physics 6 (3) (2012) 139-145. 